\title{
JAPANESE INVESTIGATIONS ON NUCLEIC ACIDS
}

$\mathrm{T}$

THE Seventh Symposium on Nucleic Acids was held at Nagoya University during February 1-2 under the auspices of the Association for Nucleic Acid Research, which was founded in 1949 and has two hundred members in Japan. The first of the Symposia on Nucleic Acids was held in Tokyo in 1949. At that time, research activities in Japan were greatly hampered by the profound economic and social difficulties of the confused post-war period, and were still in an embryonic state. Papers read before that meeting consisted mainly of review articles of work carried out in other countries. In the following years the Symposia, held at intervals in Tokyo, Kobe and Osaka, helped to stimulate the enthusiasm of Japanese scientists for this very important subject. At the Sixth Symposium (September 1956, Osaka), the meeting for the first time took the form of an international event, four papers being read by scientists from the United States who were visiting Japan.

The latest meeting was honoured by the presence of Dr. Yvonne Khouvine (Institut de Biologie physico-chimique, Paris), who read a paper which summarized the results of metabolic studies on atypical epithelioma, conducted during the past few years in her laboratory, especially in collaboration with Dr. K. Yagi (Department of Biochemistry, Nagoya University Medical School), who also attended the meeting.

In all sessions there were many papers by young workers. Each paper was followed by a discussion, which made the meeting highly informative. Altogether, fifteen papers were read before an audience totalling 60-100 people from different parts of Japan. Some of the important results presented by contributors may be briefly summarized.

By physical and chemical studies of nucleic acids, Y. Kawade (Institute for Virus Research, Kyoto University) and T. Kitamura (Institute of Science and Technology, University of Tokyo) showed that the molecular weight of ribonucleic acid from yeast and liver, prepared by hot dodecyl sulphate extraction, with guanidine hydrochloride, or with phenol, was as high as 1-3 $\times 10^{5}$ and that of tobacco mosaic virus ribonucleic acid (guanidine method) $1-2 \times 10^{6}$. The configuration of these acids in solution was markedly different from that of deoxyribonucleic acid, and the size and shape of ribonucleic acid depended to a large extent on the ionic strength and other factors. High concentrations of sodium and magnesium chlorides eventually caused precipitation of ribonucleic acid, but this was not the case with deoxyribonucleic acid. Whether these ribonucleic acids are aggregations of smaller units remains uncertain. Two highly interesting papers came from the laboratory of Prof. F. Egami, the present president of the Association. M. Naoi, K. Sato-Asano and F. Egami (Department of Chemistry, Nagoya University) determined the specificity of two ribonucleases $\left(T_{1}\right.$ and $\left.T_{2}\right)$ isolated from takadiastase. The specificity of the two enzymes, both resistant to heat, was entirely different from that of pancreatic ribonuclease I. Thus, ribonuclease $T_{1}$, with $p \mathrm{H}$ optimum $7 \cdot 5$, specifically hydrolysed phosphate diester bonds of ribonucleic acid at the $5^{\prime}$-phosphate position of guanylic acid, via $2^{\prime}, 3^{\prime}$-cyclic phosphate; ribo- nuclease $T_{2}$ had its $p H$ optimum at $4 \cdot 5$, and specifically attacked adenylic acid $5^{\prime}$-phosphate bonds in ribonucleic acid. Various aspects of the structure of ribonucleic acid and the mode of enzyme action were also presented and discussed. The hydrazinolysis of nucleic acids, leading to the preparation of ribo- and deoxyribo-apyrimidinic acid, was reported by $\mathrm{S}$. Takemura and M. Miyazaki (Department of Chemistry, Nagoya University). Deoxyriboapyrimidinic acid was subjected to the procedure of preparing apurinic acid, yielding polydeoxyribophosphate. These novel compounds were non-dialysable, though the size of the molecule was smaller than that of the original nucleic acids. The importance of this work for the study of nucleic acid structure is evident, and further progress will be rapid.

Physical and chemical studies of ribonucleoprotein particles of rabbit liver cells, reported by M. Takanami (National Institute of Animal Health), revealed the heterogeneity of the so-called microsomes. They consisted of a $250 \mathrm{~S}$. fraction rich in lipids but poor in ribonucleic acid and a $50 \mathrm{~S}$. fraction which was made up of ribonucleoprotein particles with an acid/protein ratio of $2: 3$; this accounted for $45-55$ per cent of the cytoplasmic ribonucleic acid. On treatment with deoxycholate the $250 \mathrm{~S}$. fraction liberated a labile ribonucleic acid and another ribonucleoprotein with a sedimentation rate of $50 \mathrm{~S}$. This latter could only be isolated after complete destruction of the $250 \mathrm{~S}$. fraction, and its ribonucleic acid showed a marked difference from that of the $50 \mathrm{~S}$. fraction mentioned above in the rate of uptake of phosphorus-32. Takanami emphasized that the term 'microsome' is a concept associated with the differential centrifugation technique, and may not represent any real structural entity.

On the metabolism and function of ribonucleic acid and ribonucleoprotein, S. Osawa (Biological Institute, Nagoya University) discussed investigations designed to test the hypothesis that a ribonucleic acid extracted from nuclei of animal tissue cells with $p \mathrm{H} 7 \cdot 1$ phosphate buffer might serve as the precursor of microsomal ribonucleoprotein. The findings, including analysis of nucleotide composition, electrophoretic and ultracentrifugal studies as well as tracer experiments, however, were inconclusive. It was then suggested that the incorporation of labelled precursors into microsomal ribonucleic acid was equivalent to that into deoxyribonucleic acid and represented the production of new microsome particles linked to cell proliferation. A very interesting result emerged, however, when he turned to the hypothesis that the $p H$ 7.1 ribonucleoprotein of the nucleus, because of its similarity to ribonucleoprotein obtained by the same method from microsomes, might serve as the site of nuclear protein synthesis. Thus, the alanine labelled with carbon-14 was incorporated at an exceedingly high rate into $p H$ 7.1 ribonucleoproteins of the nucleus and the microsomes, in vitro, and the label was completely separated from the ribonucleoprotein by treatment with streptomycin. K. Miura and I. Watanabe (Institute of Science and Technology, University of Tokyo) reported the nucleotide composition of ribonucleic acid synthesized immediately following phage infection, but before the onset of the synthesis of 
phage deoxyribonucleic acid and phage protein, in a $T$ phage-E. coli complex. The nucleotide composition, estimated from the incorporation of phosphorus-32 into individual nucleotides of ribonucleic acid, was unlike that of normal $E$. coli and was specific to the phage species $\left(T_{2}\right.$ and $T_{3}$ were compared). It was not affected by various conditions which prevented phage deoxyribonucleic acid synthesis or the production of progeny phage. But it corresponded fairly well to the nucleotide composition of phage deoxyribonucleic acids, the replacement of the ribonucleic acid pyrimidines for the phage deoxyribonucleic acid pyrimidines being taken into acsount. Thus, the ribonucleic acid synthesis at an early period of phage infection seems to be under direct control of phage deoxyribonucleic acid. $Y$. Hotta (Biological Institute, Nagoya University) gave a convincing proof that in the early development of fern gametophytes one-dimensional cell division (leading to the elongation of the plant) and two-dimensional division which followed (leading to the fan-like growth) depended on the amount of protein synthesis. One of the simplest forms of differentiation was thus correlated with the biochemical activity of the cell. 8-Azaguanine, which, like amino-acid analogues, specifically inhibited the two-dimensional cell division, besides being incorporated into ribonucleic acid, produced an appreciable amount of an unknown ultraviolet-absorbing material (probably a nucleotide) in an alkaline digest of ribonucleic acid.

As regards the biosynthesis of deoxyribonucleic acid, T. and R. Okazaki (Biological Institute, Nagoya University) showed that the majority of the acid. soluble deoxyribosidic compounds, found in a deoxyriboside-requiring strain of Lactobacillus acidophilus cells, were more than simple nucleosides. The main component was probably a derivative of thymidine diphosphate, and its behaviour under various conditions suggested that it might be an intermediate in deoxyribonucleic acid synthesis. It was also shown that the deoxyribonucleic acid synthesis in the same bacteria can proceed in the absence of ribonucleic acid and protein syntheses and vice versa, while ribonucleic acid synthesis and protein synthesis were inhibited together either by uracil deficiency or by amino-acid deficiency. The inter-as well as intra-molecular homogeneity of deoxyribonucleic acid, with respect to labelling with phosphorus-32, was evidenced by the experiments of Sakaki (Chemical Laboratory of General Education, Nagoya University), using rabbit appendix removed $30 \mathrm{~min}$. after injection with phosphorus-32. The role of pre-existing deoxyribonucleic acid in the incorporation of phosphorus-32 into isolated nuclei of rabbit appendix was discussed by M. Sekiguchi and A. Sibatani (Cytochemistry Laboratory, Yamaguti Medical School). Incorporation into nucleic acid was abolished by treating these nuclei with deoxyribonuclease I but could be restored by adding deoxyribonucleic acid or ribonucleic acid of heterologous origin; even the addition of chondroitin sulphate was effective in restoring the lost activity, suggesting that the effect of added substances was physico-chemical, rather than due to the specificity or the supply of energy-rich nucleotides. The role of deoxyribonucleic acid synthesis in enzyme formation was discussed by N. Otsuji and Y. Takagi (Department of Biology, Faculty of Science, and Department of Biochemistry, Medical School, Osaka University). Intact and 'shocked' protoplasts of $E$. coli strains were capable of forming tryptophanase (by induction) and deoxyribonucleic acid. The presence of 5-bromouracil and 6-azauracil effectively inhibited enzyme formation by preparations of strain $K-12$, while they had no effect when tested with intact cells. With preparations of the thymine-requiring strain $15 T^{-}$, enzyme formation and deoxyribonucleic acid synthesis depended on the presence of thymine. In the absence of thymine only a small amount of enzyme was formed at an early stage.

A limited number of the proceedings of this symposium (in English) can be obtained on request, free of eharge, from the Association for Nucleic Acid Research, c/o Prof. T. Suzuki, Department of Pharmacology, University of Kyoto Medical School, Yosida, Kyoto, Japan.
A. Sibatani

\section{SALT MARSHES}

A SMALL, representative, and successful conference on salt marshes was held at Sapelo Island, Georgia, the Marine Institute of that University, during March 24-29. It was sponsored by the National Science Foundation and the University of Georgia.

There were forty-seven members, including two from Germany, one from Holland, one from New Zealand, and one from Great Britain. All the others were from the United States and Canada. Dr. R. A. Ragotzkie and his colleagues organized both conference and excursions, and all participants are most grateful to them.

The Conference was under the presidency of Dr. A. C. Redfield of Woods Hole, and on each separate day a discussion leader was in charge of the morning session. The first day was devoted to papers on salt marshes as land forms. J. A. Steers acted as chairman and introduced the subject by a paper on the physiography of salt marshes, on both temperate and tropical coasts. L. M. van Straaten followed with an account of the origin of the tidal flats of Holland.
R. A. Ragotzkie examined marsh drainage patterns, and were followed by R. J. Russell and J. P. Morgan who described the morphology of the Louisiana marshes and the coastal changes following hurricane 'Audrey'. In the afternoon there was a field trip to the beach on Sapelo Island, and a few members made a long excursion on the mainland to see the terrace formations characteristic of the coast. In the evening J. Zeigler showed some very pertinent films, taken from the air, of large parts of the Carolina and Georgia coast.

The second day was devoted to a discussion on the vegetation of salt marshes. V. J. Chapman provided a general perspective of the vegetation and showed that maritime salt marshes can be divided into a number of different groups in the different parts of the world. The relationships within a group and between groups can be determined either by analysis of the various floral elements or else by means of affinity coefficients. After E. T. Moul had described the algal flora of salt pans on a marsh the remainder of the morning was devoted to a paper by $P$. $R$. 